Article

\title{
On Hybrid Type Nonlinear Fractional Integrodifferential Equations
}

\author{
Faten H. Damag ${ }^{1}$, Adem K1lıçman ${ }^{2, *(1)}$ and Awsan T. Al-Arioi ${ }^{1}$ \\ 1 Department of Mathematics, University Taiz, 6803 Taiz, Yemen; faten@212326@hotmail.com (F.H.D.); \\ awsan2018.t@gmail.com (A.T.A.-A.) \\ 2 Department of Mathematics and Institute for Mathematical Research, University Putra Malaysia, \\ Serdang 43400, Malaysia \\ * Correspondence: akilic@upm.edu.my
}

Received: 21 April 2020; Accepted: 18 May 2020; Published: 16 June 2020

\begin{abstract}
In this paper, we introduce and investigate a hybrid type of nonlinear Riemann Liouville fractional integro-differential equations. We develop and extend previous work on such non-fractional equations, using operator theoretical techniques, and find the approximate solutions. We prove the existence as well as the uniqueness of the corresponding approximate solutions by using hybrid fixed point theorems and provide upper and lower bounds to these solutions. Furthermore, some examples are provided, in which the general claims in the main theorems are demonstrated.
\end{abstract}

Keywords: fractional integro-differential equation; hybrid type fractional integro-differential equation; fixed point theorems; Dhage theorem; approximations solutions; Lipschitz conditions; weaker mixed partial continuity

MSC: 26A33

\section{Introduction}

Nonlinear fractional integro-differential equations are an important class of equations and widely applied in many areas such as physics, electromagnetic, mechanics, biology, signal processing, finance (in particular option pricing), economics and many more. There are also many different methods to solve these types of equations, in particular, to study the solutions of existence and uniqueness. Further properties for these types of equations have been studied by many researchers using different techniques. Sitho et al. in [1] used the couple of hybrid fixed point theorems for the sum of three operators to prove the existence solutions for this type of equations. In 2016, Dhage et al. [2] studied the existence and approximate solutions of this kind of equations by using a hybrid fixed point theorem under weaker mixed partial continuity and Lipschitz conditions. In the same year, Dhage et al. (see, [3]) introduced and proved algorithms for the existence of solution for nonlinear first order ordinary integro-differential equations and to approximate the solutions for initial value problems. In 2019, Ardjouni and Djoudi [4] proved the existence and approximation of solutions of these types of equations by using Dhage iteration principle. The hybrid equations have applications of modelling non-linear systems, hybrid systems and physical systems with fractional calculus; e.g., human factors (2019), where fractional calculus is used to model the human operator $[5,6]$ and a hybrid model for vehicle driving in [7]. 
Now consider the following initial value problem (IVP) for a hybrid type nonlinear fractional integro-differential equation (HTNFIDE)

$$
\begin{aligned}
\left(D^{\alpha} u\right)(t)+\lambda u(t) & =f\left(t, u(t), \int_{t_{0}}^{t} \frac{(t-s)^{\alpha-1}}{\Gamma(\alpha)} g(s, u(s)) d s\right) \\
u\left(t_{0}\right) & =B_{0} \in \mathbb{R} .
\end{aligned}
$$

for all $t \in J$ and for some positive real $\lambda \in \mathbb{R}$, where $f: J \times \mathbb{R} \times \mathbb{R} \rightarrow \mathbb{R}$ and $g: J \times \mathbb{R} \rightarrow \mathbb{R}$ are continuous functions, then $D^{\alpha}$ denotes the Riemann-Liouville fractional derivative of order $\alpha(0<\alpha \leq 1)$ and $J=\left[t_{0}, t_{0}+a\right]$ closed-bounded interval for $t_{0}, a \in \mathbb{R}$ with $t_{0} \geq 0$ and $a>0$. By a function $u \in C^{1}(J, \mathbb{R})$ that satisfies the above problem IVP, the soulution of Equation (1), where $C^{1}(J, \mathbb{R})$ is the space of differentiable continuous functions defined on $J$.

The present work is organized as follows: Firstly, we study the existence and uniqueness results for IVPs. Next, we give an existence result for IVPs for hybrid fractional differential equations with linear perturbations of the first type.

\section{Preliminaries}

Here, we recall some related concepts which will be useful in our study; details can be found in [8-12].

Definition 1 ([13,14]). The following operator $I^{\alpha}$ defined by

$$
I^{\alpha} h(x)=\frac{1}{\Gamma(\alpha)} \int_{a}^{x} \frac{h(\mu)}{(x-\mu)^{1-\alpha}} d \mu, \alpha>0,
$$

for $x \in[a, b]$, is said to be a Riemann-Liouville fractional integral having order $\alpha$ and similarly, the operator $D^{\alpha}$

$$
D^{\alpha} h(u)=\frac{1}{\Gamma(m-\alpha)}\left[\frac{d}{d u}\right]^{m} \int_{a}^{u} \frac{h(\mu)}{(u-\mu)^{\alpha-m+1}} d \mu,(m-1) \leq \alpha<m,
$$

is called a Riemann-Liouville fractional differential, here $m$ is an integer and $\alpha$ is a real number for $u \in[a, b]$, see the details in $[15,16]$.

Throughout this study, $E(\|\|,. \preceq)$ represents partially ordered linear normed space with respect to relation $\preceq$.

The space $E(\|\|,. \preceq)$ is called a regular ( see, [17]) if for non-increasing (resp., non-decreasing) sequence $\left\{u_{n}\right\}$ in $E$ such that $u_{n} \rightarrow u^{*}$ as $n \rightarrow \infty, u_{n} \succeq u^{*}$ (resp., $u_{n} \preceq u^{*}$ ) for all $n \in \mathbb{N}$.

Definition $2([18,19])$. A function $T: E \rightarrow E$ is said to be partially continuous at $a \in E$ for every $\epsilon>0$ if there exists a $\delta>0$ such that

$$
\|u-a\|<\delta \Rightarrow\|T u-T a\|<\epsilon,
$$

then the map $T$ is called partially continuous on whole space $E$.

Definition 3 ([20,21]). A subset $\Omega \neq \varnothing$ of the partially ordered Banach space $E$ is said to be partially compact if $B$ in $\Omega$ is a relatively compact set in $E$.

Definition $4([20,22])$. A function $T: E \rightarrow E$ is called:

1. partially compact if $T(E)$ is a partially relatively compact set in $E$,

2. uniformly partially compact if it is partially compact and uniformly partially bounded on $E$,

3. partially totally bounded if for any bounded subset $\Omega$ of $E, T(\Omega)$ is a partially relatively compact subset of $E$, 
4. partially continuous and partially totally bounded, then it is called partially completely continuous on E.

Definition 5 ([3,18]). A metric $d$ and the relation $\preceq$ on $E \neq \varnothing$ are called compatible if for every monotone sequence $\left\{u_{n}\right\}$ in $E$ with a subsequence $\left\{u_{n_{k}}\right\}$ of $\left\{u_{n}\right\}$ converges to $u^{*}$, the sequence $\left\{u_{n}\right\}$ converges to $u^{*}$. The set $\Omega \subseteq E$ is called Janhavi subset of $E$ if the metric $d$ and the relation $\preceq$ are compatible in the set $\Omega$. In particular, $E$ is called a Janhavi Banach space or Janhavi metric if $\Omega=E$.

Remark 1. Note that the set of real numbers $R$ with absolute value function as a norm and with the usual order relation $\leq$ has compatibility property. Further, each Euclidean space of finite dimensional $R^{n}$ has the same property of compatibility with respect to relationship $\preceq$ with the usual components and with the standard norm $\|\cdot\|$ in $\mathbb{R}^{n}$.

The iteration method was developed in [18,20,22], and incorporated into the following theorems, which are the main tools that will be applied to some of the problems in this work. Note that "the monotonic convergence" for successive approximations sequence to the solution of a nonlinear equation that begins with a lower or upper solution of the equations as its initial approximation". This is also a useful tool to examine the existence of a solution in nonlinear analysis. As we will see, Picard's successive iterations medhod differs from the usual Dhage iteration method.

Theorem 1 ([20]). Let $(E, \preceq,\|\|$.$) be a complete regular space respect to partially ordered then T: E \rightarrow E$ is a partially continuous, partially compact operator and increasing. If there exists an element $u_{0} \in E$ such that $u_{0} \preceq T u_{0}$ or $T u_{0} \preceq u_{0}$, then the operator $T u=u$ has a solution $u^{*}$ in $E$, and thus sequence $\left\{T^{n} u_{0}\right\}$ converges to $u^{*}$.

Definition 6. An upper semi-continuous and monotone increasing function $\xi: R^{+} \rightarrow R^{+}$is said to be a $D$-function provided that $\xi(0)=0$. Then, an operator $T: E \rightarrow E$ is said to be a partial $D$-contraction if there is a D-function satisfies

$$
\|T u-T v\| \leq \xi(\|u-v\|)
$$

for $u, v \in E$, where $0<\xi(r)<r$, for $r>0$. In particular, if $\xi(r)=k r$, for $k>0$, then $T$ is called a Lipschitz operator and if $0<k<1$, then $T$ is known as contraction on $(E, \preceq,\|\|$.$) .$

Theorem 2. Let $(E, \preceq,\|\|$.$) be a partially ordered, regular complete normalized linear space with the norm \|$.$\| .$ Further $A, B: E \rightarrow E$ be two increasing operators such that

(a) the operator $A$ is a partial nonlinear D-contraction and partially bounded,

(b) the operator $B$ is partially compact and partially continuous, and

(c) there exists an element $u_{0} \in E$ such that $u_{0} \preceq A u_{0}+B u_{0}$ or $u_{0} \succeq A u_{0}+B u_{0}$.

Then the equation $A u+B u=u$ has a solution $u^{*}$ in $(E, \preceq,\|\|$.$) and sequence u_{n+1}=A u_{n}+B u_{n}$ converges monotonically to $u^{*}$.

\section{Existence and Uniqueness of Solutions}

In this section, we first define the order relation $\preceq$ and respect to norm $\|$.$\| in C(J, \mathbb{R})$ as follows

$$
\|u\|=\sup _{t \in J}|u(t)|
$$

and

$$
u \leq v \Leftrightarrow u(t) \leq v(t), \text { for all, } t \in J
$$

Note that $C(J, \mathbb{R})$ is a Banach space with respect to supremum norm and is also partially ordered respect to $\preceq$. Also $C(J, \mathbb{R})$ is regular and a lattice, that is, each pair of elements has an upper and lower bound. 
The following lemma is an application of the Arzela-Ascoli theorem.

Lemma 1. Let $(C(J, \mathbb{R}), \preceq,\|\|$.$) be partially ordered with respect to \preceq$ and norm $\|$.$\| . Then, \preceq$ and $\|$.$\| are$ compatible in a compact subset of $C(J, \mathbb{R})$.

We can see the proof of the lemma in [3]. Next. we need the following:

Definition 7. A differentiable function $z \in C^{1}(J, \mathbb{R})$ is a lower solution for (1) if fulfills

$$
\begin{aligned}
\left(D^{\alpha} z\right)(t)+\lambda z(t) & \leq f\left(t, z(t), \int_{t_{0}}^{t} \frac{(t-s)^{\alpha-1}}{\Gamma(\alpha)} g(s, z(s))\right) d s \\
z\left(t_{0}\right) & \leq B_{0} \in \mathbb{R},
\end{aligned}
$$

for every tin J. Similarly, an upper solution $y \in C^{1}(J, \mathbb{R})$ for (1) if staisfies the inverting the inequalities in (6).

\subsection{Existence Theorem}

For the future discussion we need the following assumptions: Let

$\left(A_{1}\right)$ there is a constant $M_{f}>0$ such that $|f(t, u, v)| \leq M_{f}, \forall t \in J$ and $u$ in $\mathbb{R}$.

$\left(A_{2}\right)$ the function $f(t, u, v)$ is monotone increasing in $u$ and $v$ for any $t$ in $J$.

$\left(A_{3}\right)$ the function $g(t, u)$ is monotone increasing in $u$ for any $t$ in $J$.

$\left(A_{4}\right)$ the (1) has a lower solution $z \in C^{1}(J, \mathbb{R})$.

$\left(A_{5}\right)$ there exists a constant $L>0$ such that

$$
0 \leq g(t, u)-g(t, v) \leq L(u-v)
$$

for every $t \in J, \quad u, v \in \mathbb{R}$, with $u \geq v$.

$\left(A_{6}\right)$ There are $\mathrm{D}-$ functions $\xi_{1}$ and $\xi_{2}$ such that

$$
0 \leq f\left(t, x_{1}, x_{2}\right)-f\left(t, y_{1}, y_{2}\right) \leq \xi_{1}\left(x_{1}-y_{1}\right)+\xi_{2}\left(x_{2}-y_{2}\right)
$$

for all $u_{1}, u_{2}, v_{1}, v_{2} \in \mathbb{R}$ with $u_{1} \geq v_{1}$ and $u_{2} \geq v_{2}$. Furthermore

$$
\frac{a^{\alpha}}{\Gamma(\alpha+1)}\left(\xi_{1}(r)+\xi_{2}(\text { Lar })\right)<r, \text { for any, } r>0
$$

$\left(A_{7}\right)$ The the Equation (18) has a lower solution $z \in C^{1}(J, \mathbb{R})$.

The following lemma is important in our proof and related to Riemann \& Liouville differential operator.

Lemma 2. Let $h: J \rightarrow \mathbb{R}$, be an integrable function then $u \in C^{1}(J, \mathbb{R})$ is a solution for (1)

$$
\begin{aligned}
\left(D^{\alpha} u\right)(t)+\lambda u(t) & =h(t), \quad t \in J \\
u\left(t_{0}\right) & =B_{0} \in \mathbb{R}
\end{aligned}
$$

if and only if

$$
u(t)=c e^{-\lambda \frac{t^{\alpha}}{\alpha !}}+e^{-\lambda \frac{t^{\alpha}}{\alpha !}} \int_{t_{0}}^{t} \frac{(t-s)^{\alpha-1}}{\Gamma(\alpha)} e^{\lambda s^{\alpha^{\alpha}}} h(s) d s, \quad t \in J
$$

where $c=B_{0} e^{\frac{\lambda t_{0}^{\alpha}}{\Gamma(\alpha)}}$ is a constant.

Next we have 
Theorem 3. Let the assumptions $\left(A_{1}-A_{4}\right)$ be held. Then the HTNFIDE (4) has a solution $u^{*}$ on J and the sequence $\left\{u_{n}\right\}_{n=1}^{\infty}$ satisfies

$$
\begin{aligned}
u_{1}(t) & =y(t), \\
u_{n+1}(t) & =c e^{-\lambda \frac{t^{\alpha}}{\alpha !}} \\
& +e^{-\lambda \frac{t^{\alpha}}{\alpha !}} \int_{t_{0}}^{t} \frac{(t-s)^{\alpha-1}}{\Gamma(\alpha)} e^{\lambda \frac{s^{\alpha}}{\alpha !}} f\left(s, u_{n}(s), \int_{t_{0}}^{s} \frac{(s-\xi)^{\alpha-1}}{\Gamma(\alpha)} g\left(\xi, u_{n}(\xi)\right) d \xi\right) d s
\end{aligned}
$$

for all $t$ in $\mathbb{R}$, converges to $u^{*}$.

Proof. From Lemma 2, the HTNFIDE (4) is equivalent to the nonlinear integral equation

$$
\begin{aligned}
u(t) & =c e^{-\lambda \frac{t^{\alpha}}{\alpha !}} \\
& +e^{-\lambda \frac{t^{\alpha}}{\alpha !}} \int_{t_{0}}^{t} \frac{(t-s)^{\alpha-1}}{\Gamma(\alpha)} e^{\lambda \frac{s^{\alpha}}{\alpha !}} f\left(s, u(s), \int_{t_{0}}^{s} \frac{(s-\xi)^{\alpha-1}}{\Gamma(\alpha)} g(\xi, u(\xi)) d \xi\right) d s .
\end{aligned}
$$

Set $E=C(J, \mathbb{R})$. Then, by Lemma 1 it follows that each compact chain in $E$ has the property of compatibility with respect to the order relation $\preceq$ and the norm $\|$.$\| in E$. We introduce the operator $T$ by

$$
T u(t)=e^{-\lambda \frac{t^{\alpha}}{\alpha !}}\left(c+\int_{t_{0}}^{t} \frac{(t-s)^{\alpha-1}}{\Gamma(\alpha)} e^{\lambda \frac{s^{\alpha}}{\alpha !}} f\left(s, u(s), \int_{t_{0}}^{s} \frac{(s-\xi)^{\alpha-1}}{\Gamma(\alpha)} g(\xi, u(\xi)) d \xi\right) d s\right)
$$

Since $T u$ is continuous, then $T u \in E$. That is, $T$ maps E into itself. The HTNFIDE (4) is then equivalent to the operator equation

$$
T u(t)=u(t), \quad t \in J .
$$

Through a series of steps, we must prove that the operator $T$ fulfills all the conditions of Theorem 1.

Step I: Let $T$ be increasing on $E$ and for $u, v$ in $E$ with $u \leq v$. Then, from $\left(A_{2}\right)$, we get

$$
\begin{aligned}
T u(t) & =e^{-\lambda \frac{t^{\alpha}}{\alpha !}}\left(c+\int_{t_{0}}^{t} \frac{(t-s)^{\alpha-1}}{\Gamma(\alpha)} e^{\lambda \frac{s^{\alpha}}{\alpha !}} f\left(s, u(s), \int_{t_{0}}^{s} \frac{(s-\xi)^{\alpha-1}}{\Gamma(\alpha)} g(\xi, u(\xi)) d T\right) d s\right) \\
& \leq e^{-\lambda \frac{t^{\alpha}}{\alpha !}}\left(c+\int_{t_{0}}^{t} \frac{(t-s)^{\alpha-1}}{\Gamma(\alpha)} e^{\lambda \frac{s^{\alpha}}{\alpha !}} f\left(s, v(s), \int_{t_{0}}^{s} \frac{(s-\xi)^{\alpha-1}}{\Gamma(\alpha)} g(\xi, v(\xi)) d \xi\right) d s\right) \\
& =T v(t)
\end{aligned}
$$

for all $t$ in $J$. This proves that $T$ is a increasing operator on $E$.

Step II: Let $T$ be partially continuous on $E .\left\{u_{n}\right\}$ is a chain points sequences $C$ in $E$ such that $u_{n} \rightarrow u, \forall n \in N$. Then, by the controlled convergence theorem,

$$
\begin{aligned}
& \lim _{n \rightarrow \infty} T u_{n}(t)= \\
& \lim _{n \rightarrow \infty}\left(e^{-\lambda \frac{t^{\alpha}}{\alpha !}}\left(c+\int_{t_{0}}^{t} \frac{(t-s)^{\alpha-1}}{\Gamma(\alpha)} e^{\lambda \frac{s^{\alpha}}{\alpha !}} f\left(s, u_{n}(s), \int_{t_{0}}^{s} \frac{(s-\xi)^{\alpha-1}}{\Gamma(\alpha)} g\left(\xi, u_{n}(\xi)\right) d \xi\right) d s\right)\right) \\
= & e^{-\lambda \frac{t^{\alpha}}{\alpha !}}\left(c+\int_{t_{0}}^{t} \frac{(t-s)^{\alpha-1}}{\Gamma(\alpha)} e^{\lambda \frac{s^{\alpha}}{\alpha !}} f\left(s, u(s), \int_{t_{0}}^{s} \frac{(s-\xi)^{\alpha-1}}{\Gamma(\alpha)} g(\xi, u(\xi)) d \xi\right) d s\right) \\
= & T u(t)
\end{aligned}
$$


for every $t$ in $J$. This proves that $\left\{T u_{n}\right\}$ converges to $T u$ point-wise on $J$. Therefore, we prove that $\left\{T u_{n}\right\}$ is an equi-continuous sequence of functions in $E$. Let $t_{1}, t_{2}$ in $J$ and $t_{1}<t_{2}$. Then

$$
\begin{aligned}
& \left|T u_{n}\left(t_{2}\right)-T u_{n}\left(t_{1}\right)\right| \leq c\left|e^{-\lambda \frac{t_{1}^{\alpha}}{\alpha !}}-e^{-\lambda \frac{t_{2}^{\alpha}}{\alpha !}}\right| \\
& +\mid e^{-\lambda \frac{t_{2}^{\alpha}}{\alpha !}} \int_{t_{0}}^{t_{2}} \frac{\left(t_{2}-s\right)^{\alpha-1}}{\Gamma(\alpha)} e^{\lambda \frac{s^{\alpha}}{\alpha !}} f\left(s, u_{n}(s), \int_{t_{0}}^{s} \frac{(s-\xi)^{\alpha-1}}{\Gamma(\alpha)} g\left(\xi, u_{n}(\xi)\right) d \xi\right) d s \\
& -e^{-\lambda \frac{t_{1}^{\alpha}}{\alpha !}} \int_{t_{0}}^{t_{1}} \frac{\left(t_{1}-s\right)^{\alpha-1}}{\Gamma(\alpha)} e^{\lambda \frac{s^{\alpha}}{\alpha !}} f\left(s, u_{n}(s), \int_{t_{0}}^{s} \frac{(s-\xi)^{\alpha-1}}{\Gamma(\alpha)} g\left(\xi, u_{n}(\xi)\right) d \xi\right) d s \mid \\
& \leq c\left|e^{-\lambda \frac{t_{1}^{\alpha}}{\alpha !}}-e^{-\lambda \frac{t_{2}^{\alpha}}{\alpha !}}\right| \\
& +\mid e^{-\lambda \frac{t_{2}^{\alpha}}{\alpha !}} \int_{t_{0}}^{t_{2}} \frac{\left(t_{2}-s\right)^{\alpha-1}}{\Gamma(\alpha)} e^{\lambda \frac{s^{\alpha}}{\alpha !}} f\left(s, u_{n}(s), \int_{t_{0}}^{s} \frac{(s-\xi)^{\alpha-1}}{\Gamma(\alpha)} g\left(\xi, u_{n}(\xi)\right) d \xi\right) d s \\
& -e^{-\lambda \frac{t_{1}^{\alpha}}{\alpha !}} \int_{t_{0}}^{t_{2}} \frac{\left(t_{1}-s\right)^{\alpha-1}}{\Gamma(\alpha)} e^{\lambda \frac{s^{\alpha}}{\alpha !}} f\left(s, u_{n}(s), \int_{t_{0}}^{s} \frac{(s-\xi)^{\alpha-1}}{\Gamma(\alpha)} g\left(\xi, u_{n}(\xi)\right) d \xi\right) d s \mid \\
& +\mid e^{-\lambda \frac{t_{1}^{\alpha}}{\alpha !}} \int_{t_{0}}^{t_{2}} \frac{\left(t_{1}-s\right)^{\alpha-1}}{\Gamma(\alpha)} e^{\lambda \frac{s^{\alpha}}{\alpha !}} f\left(s, u_{n}(s), \int_{t_{0}}^{s} \frac{(s-\xi)^{\alpha-1}}{\Gamma(\alpha)} g\left(\xi, u_{n}(\xi)\right) d \xi\right) d s \\
& -e^{-\lambda \frac{t_{1}^{\alpha}}{\alpha !}} \int_{t_{0}}^{t_{1}} \frac{\left(t_{1}-s\right)^{\alpha-1}}{\Gamma(\alpha)} e^{\lambda \frac{s^{\alpha}}{\alpha !}} f\left(s, u_{n}(s), \int_{t_{0}}^{s} \frac{(s-\xi)^{\alpha-1}}{\Gamma(\alpha)} g\left(\xi, u_{n}(\xi)\right) d \xi\right) d s \mid \\
& \leq c\left|e^{-\lambda \frac{t_{1}^{\alpha}}{\alpha !}}-e^{-\lambda \frac{t_{2}^{\alpha}}{\alpha !}}\right|+\left|e^{-\lambda \frac{t_{2}^{\alpha}}{\alpha !}}-e^{-\lambda \frac{t_{1}^{\alpha}}{\alpha !}}\right| \\
& \times\left|\int_{t_{0}}^{t_{2}} \frac{\left(t_{2}-s\right)^{\alpha-1}-\left(t_{1}-s\right)^{\alpha-1}}{\Gamma(\alpha)} e^{\lambda s^{\alpha}} f\left(s, u_{n}(s), \int_{t_{0}}^{s} \frac{(s-\xi)^{\alpha-1}}{\Gamma(\alpha)} g\left(\xi, u_{n}(\xi)\right) d \xi\right) d s\right| \\
& +e^{-\lambda \frac{t_{1}^{\alpha}}{\alpha !} \mid} \int_{t_{0}}^{t_{2}} \frac{\left(t_{1}-s\right)^{\alpha-1}}{\Gamma(\alpha)} e^{\lambda \frac{s^{\alpha}}{\alpha !}} f\left(s, u_{n}(s), \int_{t_{0}}^{s} \frac{(s-\xi)^{\alpha-1}}{\Gamma(\alpha)} g\left(\xi, x_{n}(\xi)\right) d \xi\right) d s \\
& -\int_{t_{0}}^{t_{1}} \frac{\left(t_{1}-s\right)^{\alpha-1}}{\Gamma(\alpha)} e^{\lambda s^{\alpha}} f\left(s, u_{n}(s), \int_{t_{0}}^{s} \frac{(s-\xi)^{\alpha-1}}{\Gamma(\alpha)} g\left(\xi, u_{n}(\xi)\right) d \xi\right) d s \mid \\
& \leq c\left|e^{-\lambda \frac{t_{1}^{\alpha}}{\alpha !}}-e^{-\lambda \frac{t_{2}^{\alpha}}{\alpha !}}\right|+\left|e^{-\lambda \frac{t_{2}^{\alpha}}{\alpha !}}-e^{-\lambda \frac{t_{1}^{\alpha}}{\alpha !}}\right| \\
& \left|\int_{t_{0}}^{t_{0}+a} \frac{\left(t_{2}-s\right)^{\alpha-1}-\left(t_{1}-s\right)^{\alpha-1}}{\Gamma(\alpha)} e^{\lambda s^{s^{\alpha}}} f\left(s, u_{n}(s), \int_{t_{0}}^{s} \frac{(s-\xi)^{\alpha-1}}{\Gamma(\alpha)} g\left(\xi, u_{n}(\xi)\right) d \xi\right) d s\right| \\
& +\left|\int_{t_{1}}^{t_{2}} \frac{\left(t_{1}-s\right)^{\alpha-1}}{\Gamma(\alpha)} e^{\lambda^{\frac{s^{\alpha}}{\alpha !}}} f\left(s, u_{n}(s), \int_{t_{0}}^{s} \frac{(s-\xi)^{\alpha-1}}{\Gamma(\alpha)} g\left(\xi, u_{n}(\xi)\right) d \xi\right) d s\right| \\
& \leq\left|e^{-\lambda \frac{t_{1}^{\alpha}}{\alpha !}}-e^{-\lambda \frac{t_{2}^{\alpha}}{\alpha !}}\right|\left(c+\frac{M_{f}}{\Gamma(\alpha)} \int_{t_{0}}^{t_{0}+a}\left(t_{2}-s\right)^{(\alpha-1)}-\left(t_{1}-s\right)^{\alpha-1} e^{\lambda \frac{s^{\alpha}}{\alpha !}} d s\right) \\
& +\frac{M_{f}}{\Gamma(\alpha)}\left|\int_{t_{1}}^{t_{2}}-\left(t_{1}-s\right)^{\alpha-1} e^{\lambda \frac{s^{\alpha}}{\alpha !}}\right| d s \\
& \leq\left|e^{-\lambda \frac{t_{1}^{\alpha}}{\alpha !}}-e^{-\lambda \frac{t_{2}^{\alpha}}{\alpha !}}\right|\left(c+\frac{M_{f}}{\Gamma(\alpha)} \int_{t_{0}}^{t_{0}+a}\left(\left(t_{2}-s\right)^{(\alpha-1)}-\left(t_{1}-s\right)^{\alpha-1}\right) e^{\lambda \frac{s^{\alpha}}{\alpha !}} d s\right) \\
& +\frac{M_{f}}{\Gamma(\alpha)} \mid\left(p\left(t_{1}\right)-p\left(t_{2}\right) \mid\right. \\
& \leq\left|e^{-\lambda \frac{t_{1}^{\alpha}}{\alpha !}}-e^{-\lambda \frac{t_{2}^{\alpha}}{\alpha !}}\right|\left(c+\frac{M_{f} \cdot e^{\lambda\left(t_{0}+a\right)^{\alpha-1}}}{\Gamma(\alpha)}\right)+\frac{M_{f}}{\Gamma(\alpha)} \mid\left(p\left(t_{1}\right)-p\left(t_{2}\right) \mid\right.
\end{aligned}
$$


where, $p(t)=\int_{t_{0}}^{t} \frac{\left(t_{0}+a-s\right)^{\alpha-1}}{\Gamma(\alpha)} e^{\lambda s^{\frac{s^{\alpha}}{\alpha !}}} d s, \quad t \in J$.

Step III: Let $T$ be a operator of partially compact on $E$ and $C$ an arbitrary chain in $E$. Then we prove that $T(C)$ is a equi-continuous an uniformly bounded set in $E$. Firstly, we prove that $T(C)$ is uniformly bounded. We put $u \in C$ is arbitrary. Then,

$$
\begin{aligned}
&|T u(t)| \leq\left|c e^{-\lambda \frac{t^{\alpha}}{\alpha !}}\right| \\
&+\left|e^{-\lambda \frac{t^{\alpha}}{\alpha !}} \int_{t_{0}}^{t} \frac{(t-s)^{\alpha-1}}{\Gamma(\alpha)} e^{\lambda \frac{s^{\alpha}}{\alpha !}} f\left(s, u(s), \int_{t_{0}}^{s} \frac{(s-\xi)^{\alpha-1}}{\Gamma(\alpha)} g(\xi, u(\xi)) d \xi\right) d s\right| \\
& \leq|c|+\frac{a^{\alpha} M_{f}}{\Gamma(\alpha+1)}=r
\end{aligned}
$$

for every $t$ in $J$. We are taking the supremum over $t$, we get $\|T u\| \leq r$ for every $u \in C$. Thus $T(C) \prec E$ is a uniformly bounded. Therefore, we will prove that $T(C)$ is an equicontinuous set in $E$. We put $t_{1}, t_{2} \in J$ is arbitrary with $t_{1}<t_{2}$. Then

$$
\begin{aligned}
& \left|T u\left(t_{2}\right)-T u\left(t_{1}\right)\right| \leq c\left|e^{-\lambda \frac{t_{1}^{\alpha}}{\alpha !}}-e^{-\lambda \frac{t_{2}^{\alpha}}{\alpha !}}\right| \\
& +\mid e^{-\lambda \frac{t_{2}^{\alpha}}{\alpha !}} \int_{t_{0}}^{t_{2}} \frac{\left(t_{2}-s\right)^{\alpha-1}}{\Gamma(\alpha)} e^{\lambda s} f\left(s, u(s), \int_{t_{0}}^{s} \frac{(s-\xi)^{\alpha-1}}{\Gamma(\alpha)} g(\xi, u(\xi)) d \xi\right) d s \\
& -e^{-\lambda \frac{t_{1}^{\alpha}}{\alpha !}} \int_{t_{0}}^{t_{1}} \frac{\left(t_{1}-s\right)^{\alpha-1}}{\Gamma(\alpha)} e^{\lambda \frac{s^{\alpha}}{\alpha !}} f\left(s, u(s), \int_{t_{0}}^{s} \frac{(s-\xi)^{\alpha-1}}{\Gamma(\alpha)} g(\xi, u(\xi)) d \xi\right) d s \mid \\
& \leq c\left|e^{-\lambda \frac{t_{1}^{\alpha}}{\alpha !}}-e^{-\lambda \frac{t_{2}^{\alpha}}{\alpha !}}\right| \\
& +\mid e^{-\lambda \frac{t_{2}^{\alpha}}{\alpha !}} \int_{t_{0}}^{t_{2}} \frac{\left(t_{2}-s\right)^{\alpha-1}}{\Gamma(\alpha)} e^{\lambda \frac{s^{\alpha}}{\alpha !}} f\left(s, u(s), \int_{t_{0}}^{s} \frac{(s-\xi)^{\alpha-1}}{\Gamma(\alpha)} g(\xi, u(\xi)) d \xi\right) d s \\
& -e^{-\lambda \frac{t_{1}^{\alpha}}{\alpha !}} \int_{t_{0}}^{t_{2}} \frac{\left(t_{1}-s\right)^{\alpha-1}}{\Gamma(\alpha)} e^{\lambda \frac{s}{\alpha !}} f\left(s, u(s), \int_{t_{0}}^{s} \frac{(s-\xi)^{\alpha-1}}{\Gamma(\alpha)} g(\xi, u(\xi)) d \xi\right) d s \mid \\
& +\mid e^{-\lambda \frac{t_{1}^{\alpha}}{\alpha !}} \int_{t_{0}}^{t_{2}} \frac{\left(t_{1}-s\right)^{\alpha-1}}{\Gamma(\alpha)} e^{\lambda \frac{s^{\alpha}}{\alpha !}} f\left(s, u(s), \int_{t_{0}}^{s} \frac{(s-\xi)^{\alpha-1}}{\Gamma(\alpha)} g(\xi, u(\xi)) d \xi\right) d s \\
& -e^{-\lambda \frac{t_{1}^{\alpha}}{\alpha !}} \int_{t_{0}}^{t_{1}} \frac{\left(t_{1}-s\right)^{\alpha-1}}{\Gamma(\alpha)} e^{\lambda \frac{s}{\alpha !}} f\left(s, u(s), \int_{t_{0}}^{s} \frac{(s-\xi)^{\alpha-1}}{\Gamma(\alpha)} g(\xi, u(\xi)) d \xi\right) d s \mid \\
& \leq c\left|e^{-\lambda \frac{t_{1}^{\alpha}}{\alpha !}}-e^{-\lambda \frac{t_{l}^{\alpha}}{\alpha !}}\right|+\left|e^{-\lambda \frac{t_{2}^{\alpha}}{\alpha !}}-e^{-\lambda \frac{t_{1}^{\alpha}}{\alpha !}}\right| \\
& \times\left|\int_{t_{0}}^{t_{2}} \frac{\left(t_{2}-s\right)^{\alpha-1}-\left(t_{1}-s\right)^{\alpha-1}}{\Gamma(\alpha)} e^{\lambda s^{\alpha} !} f\left(s, u(s), \int_{t_{0}}^{s} \frac{(s-\xi)^{\alpha-1}}{\Gamma(\alpha)} g(\xi, u(\xi)) d \xi\right) d s\right| \\
& +e^{-\lambda \frac{t_{1}^{\alpha}}{\alpha !} \mid} \int_{t_{0}}^{t_{2}} \frac{\left(t_{1}-s\right)^{\alpha-1}}{\Gamma(\alpha)} e^{\lambda s^{\frac{s^{\alpha}}{\alpha !}}} f\left(s, u(s), \int_{t_{0}}^{s} \frac{(s-\xi)^{\alpha-1}}{\Gamma(\alpha)} g(\xi, u(\xi)) d \xi\right) d s \\
& -\int_{t_{0}}^{t_{1}} \frac{\left(t_{1}-s\right)^{\alpha-1}}{\Gamma(\alpha)} e^{\lambda \frac{s^{\alpha}}{\alpha !}} f\left(s, u(s), \int_{t_{0}}^{s} \frac{(s-\xi)^{\alpha-1}}{\Gamma(\alpha)} g(\xi, u(\xi)) d \xi\right) d s \mid \\
& \leq c\left|e^{-\lambda \frac{t_{1}^{\alpha}}{\alpha !}}-e^{-\lambda \frac{t_{2}^{\alpha}}{\alpha !}}\right|+\left|e^{-\lambda \frac{t_{2}^{\alpha}}{\alpha !}}-e^{-\lambda \frac{t_{1}^{\alpha}}{\alpha !}}\right| \\
& \left|\int_{t_{0}}^{t_{0}+a} \frac{\left(t_{2}-s\right)^{\alpha-1}-\left(t_{1}-s\right)^{\alpha-1}}{\Gamma(\alpha)} e^{\lambda s^{\alpha} !} f\left(s, u(s), \int_{t_{0}}^{s} \frac{(s-\xi)^{\alpha-1}}{\Gamma(\alpha)} g(\xi, u(\xi)) d \xi\right) d s\right| \\
& \left|\int_{t_{1}}^{t_{2}} \frac{\left(t_{1}-s\right)^{\alpha-1}}{\Gamma(\alpha)} e^{\lambda \frac{s^{\alpha}}{\alpha !}} f\left(s, u(s), \int_{t_{0}}^{s} \frac{(s-\xi)^{\alpha-1}}{\Gamma(\alpha)} g(\xi, u(\xi)) d \xi\right) d s\right|
\end{aligned}
$$




$$
\begin{aligned}
& \leq\left|e^{-\lambda \frac{t_{1}^{\alpha}}{\alpha !}}-e^{-\lambda \frac{t_{2}^{\alpha}}{\alpha !}}\right|\left(c+\frac{M_{f}}{\Gamma(\alpha)} \int_{t_{0}}^{t_{0}+a}\left(t_{2}-s\right)^{(\alpha-1)}-\left(t_{1}-s\right)^{\alpha-1} e^{\lambda \frac{s^{\alpha}}{\alpha !}} d s\right) \\
& +\frac{M_{f}}{\Gamma(\alpha)} \int_{t_{1}}^{t_{2}}\left(t_{1}-s\right)^{\alpha-1} e^{\lambda \frac{s^{\alpha}}{\alpha !}} d s \\
& \leq\left|e^{-\lambda \frac{t_{1}^{\alpha}}{\alpha !}}-e^{-\lambda \frac{t_{2}^{\alpha}}{\alpha !}}\right|\left(c+\frac{M_{f}}{\Gamma(\alpha)} \int_{t_{0}}^{t_{0}+a}\left(c\left(t_{2}-s\right)^{(\alpha-1)}-\left(t_{1}-s\right)^{\alpha-1}\right) e^{\lambda \frac{s^{\alpha}}{\alpha !}} d s\right) \\
& +\frac{M_{f}}{\Gamma(\alpha)} \mid\left(p\left(t_{1}\right)-p\left(t_{2}\right) \mid\right.
\end{aligned}
$$

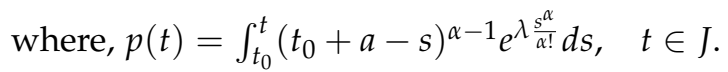

Since the functions $t \rightarrow(t-s)^{\alpha-1}$ and $t \rightarrow p(t)$ are uniformly continuous on compact interval $J=\left[t_{0}, t_{0}+a\right]$, then we have that $\left|T u\left(t_{2}\right)-T u\left(t_{1}\right)\right| \rightarrow 0$ as $t_{2} \rightarrow t_{1}$, uniformly for every $u \in C$. This proves that $T(C)$ is an equi-continuous set in $E$. Thus $T(C) \prec E$ is compact and consequently $T$ is an partially compact operator on $E \rightarrow E$.

Step IV: $z$ fulfills the operator inequality $z \leq T z$.

from the assumption $\left(A_{4}\right)$ we can obtain, $y$ is a lower solution of HTNFIDE in (4) introduced on J. Thus

$$
\left(D^{\alpha} z\right)(t)+\lambda z(t) \leq f\left(t, z(t), \int_{t_{0}}^{t} \frac{(t-s)^{\alpha-1}}{\Gamma(\alpha)} g(s, z(s)) d s\right)
$$

and

$$
z\left(t_{0}\right) \leq B_{0} \in \mathbb{R}
$$

for every $t$ in $J$. Now integrating the Equation (11) from $t_{0}$ to $t$, we have

$$
z(t) \leq c e^{-\lambda \frac{t^{\alpha}}{\alpha !}}+e^{-\lambda \frac{t^{\alpha}}{\alpha !}} \int_{t_{0}}^{t} \frac{(t-s)^{\alpha-1}}{\Gamma(\alpha)} e^{\lambda \frac{s^{\alpha}}{\alpha !}} f\left(s, z(s), \int_{t_{0}}^{s} \frac{(s-\xi)^{\alpha-1}}{\Gamma(\alpha)} g(\xi, z(\xi)) d \xi\right) d s
$$

for every $t$ in $J$. This proves that $z$ is a lower solution of the operator in equation $u=T u$.

Thus $T$ satisfies all the conditions of Theorem 1, and the Remark 1 and we can deduce the operator in equation $T u=u$ that contains the solution. Therefore, the integral equation and HTNFIDE (4) have a solution $u^{*}$ introduced in $J$. Moreover, the sequence $\left\{u_{n}\right\}$ of successive approximations introduced by Equation (7) converges monotonically to $u^{*}$. Thus we have completed the proof.

Remark 2. The inference of theorem 3 also still true if we substitute condition $\left(A_{4}\right)$ with $\left(B_{4}\right)$ The HTNFIDE (4) contain a upper solution $v \in C^{1}(J, \mathbb{R})$.

Example 1. Let $J=[0,1]$ be a closed and bounded interval, then we consider the HTNFIDE

$$
\begin{gathered}
\left(D^{\frac{3}{2}} u\right)(t)+u(t)=\tanh (u(t))+\tanh \left(\int_{0}^{t} \frac{(t-s)^{\frac{1}{2}}}{\Gamma\left(\frac{3}{2}\right)} \sinh u(s) d s\right), \\
u(0)=1 .
\end{gathered}
$$

Now if we apply the Theorem 3, we will get $\lambda=1, c=1, g(t, u)=\sinh u$ and $f(t, u, v)=$ $\tanh u+\tanh v$. Obviously, the functions $f$ and $g$ are continuous on $J \times \mathbb{R}$, and $f$ achieves $\left(A_{1}\right)$ with $M_{f}=2$. Furthermore, $g(t, u)$ is increasing in $u$ for any $t$ in $J$, and $f(t, u, v)$ is increasing in $u$ and $v$ for any $t$ in $J$, thus conditions $\left(A_{2}\right)$ and $\left(A_{3}\right)$ are achieved. Finally, the HTNFIDE (14) has a lower solution $z$ defined by

$$
z(t)=\frac{2}{\Gamma\left(\frac{3}{2}\right)}\left(e^{\frac{-t^{\frac{1}{2}}}{\Gamma\left(\frac{3}{2}\right)}}-1\right)
$$


on $J$. Hence, all the assumption of Theorem 1 are achieved, and thus HTNFIDE (14) has a solution $u^{*}$ introduced on $J$, and the sequence $\left\{u_{n}\right\}$, introduced by

$$
\begin{aligned}
& u_{1}(t)=y(t), \\
& u_{n+1}(t)=e^{-\frac{t^{\alpha}}{\alpha !}}+e^{-\frac{t^{\alpha}}{\alpha !}} \int_{0}^{t} \frac{(t-s)^{\frac{1}{2}}}{\Gamma\left(\frac{3}{2}\right)} e^{\frac{s^{\alpha}}{\alpha !}} \tanh u_{n}(s) d s \\
& +e^{-\frac{t^{\alpha}}{\alpha !}} \int_{0}^{t} \frac{(t-s)^{\frac{1}{2}}}{\Gamma\left(\frac{3}{2}\right)} e^{\frac{s^{\alpha}}{\alpha !}} \tanh \left(\int_{0}^{s} \frac{(s-\xi)^{\frac{1}{2}}}{\Gamma\left(\frac{3}{2}\right)} \sinh u_{n}(\xi) d \xi\right) d s
\end{aligned}
$$

for all $t \in J$ and where $\Gamma\left(\frac{3}{2}\right)=\frac{3 \sqrt{\pi}}{2}$, converges monotonically to $u^{*}$.

\subsection{Uniqueness Theorem}

In this section, we investigate a uniqueness theorem for the HTNFIDE (4) by using the weaker partially Lipschitz condition.

Theorem 4. A suppose that conditions $\left(A_{4}\right)-\left(A_{6}\right)$ achieve. Then the HTNFIDE (4) contain of an unique solution $u^{*}$ introduced on $J$, and the sequence $\left\{u_{n}\right\}$ of successive approximations introduced by the Equation (7) converges monotonically to $u^{*}$.

Proof. Set $E=C(J, \mathbb{R})$. Obviously, $E$ is a lattice with respect to the order relation $\preceq$ and thus upper and lower bounds there is for all pair of elements in $E$. We introduce the operator $T$ by (9). Then, the HTNFIDE (4) is equivalent to the operator Equation (10). We must prove that $T$ fulfills all the conditions of Theorem 1 .

Obviously, $T$ is an increasing operator from $E \rightarrow E$. We want to prove that the operator $T$ is a partially nonlinear $\mathrm{D}$-contraction on $E$, thus let $u, v \in E$ with $u \geq v$. Then, by $\left(A_{5}\right)$ and $\left(A_{6}\right)$

$$
\begin{aligned}
& |T u(t)-T v(t)| \leq \mid e^{-\lambda \frac{t^{\alpha}}{\alpha !}} \int_{t_{0}}^{t} \frac{(t-s)^{\alpha-1}}{\Gamma(\alpha)} e^{\lambda \frac{s^{\alpha}}{\alpha !}} f\left(s, u(s), \int_{t_{0}}^{s} \frac{(s-\xi)^{\alpha-1}}{\Gamma(\alpha)} g(\xi, u(\xi)) d \xi\right) d s \\
& -e^{-\lambda \frac{t^{\alpha}}{\alpha !}} \int_{t_{0}}^{t} \frac{(t-s)^{\alpha-1}}{\Gamma(\alpha)} e^{\lambda \frac{s^{\alpha}}{\alpha !}} f\left(s, v(s), \int_{t_{0}}^{s} \frac{(s-\xi)^{\alpha-1}}{\Gamma(\alpha)} g(\xi, v(\xi)) d \xi\right) d s \mid \\
& \leq e^{-\lambda \frac{t^{\alpha}}{\alpha !}} \int_{t_{0}}^{t} \frac{(t-s)^{\alpha-1}}{\Gamma(\alpha)} e^{\lambda \frac{s^{\alpha}}{\alpha !}} \mid f\left(s, u(s), \int_{t_{0}}^{s} \frac{(s-\xi)^{\alpha-1}}{\Gamma(\alpha)} g(\xi, u(\xi)) d \xi\right) \\
& -f\left(s, v(s), \int_{t_{0}}^{s} \frac{(s-\xi)^{\alpha-1}}{\Gamma(\alpha)} g(\xi, v(\xi)) d \xi\right) \mid d s \\
& \leq e^{-\lambda \frac{t^{\alpha}}{\alpha !}} \int_{t_{0}}^{t} \frac{(t-s)^{\alpha-1}}{\Gamma(\alpha)} e^{\lambda \frac{s^{\alpha}}{\alpha !}}\left(\xi_{1}(u(s)-v(t))\right. \\
& \left.+\xi_{2}\left(c \int_{t_{0}}^{s} \frac{(s-\xi)^{\alpha-1}}{\Gamma(\alpha)} g(\xi, u(\xi)) d \xi-g(\xi, v(\xi))\right) d \xi\right) d s \\
& \leq e^{-\lambda \frac{t^{\alpha}}{\alpha !}} \int_{t_{0}}^{t} \frac{(t-s)^{\alpha-1}}{\Gamma(\alpha)} e^{\frac{-\lambda}{\alpha !}\left(t^{\alpha}-s^{\alpha}\right)}\left(\xi_{1}(u(s)-v(s))\right. \\
& \left.+\xi_{2} \int_{t_{0}}^{s} \frac{(s-\xi)^{\alpha-1}}{\Gamma(\alpha)} L(u(\xi)-v(\xi)) d \xi\right) d s \\
& \leq \frac{1}{\Gamma(\alpha)} \int_{t_{0}}^{t}(t-s)^{\alpha-1} e^{\frac{-\lambda}{\alpha !}}\left(t^{\alpha}-s^{\alpha}\right)\left(\xi_{1}(\|u-v\|)+\xi_{2} \frac{b^{\alpha}}{\Gamma(\alpha+1)} L a\|u-v\|\right) d s \\
& \leq \xi\|u-v\|
\end{aligned}
$$


for every $t \in J$, where $\xi(r)=\frac{a^{\alpha}}{\Gamma(\alpha+1)}\left(\xi_{1}(r)+\xi_{2}(\right.$ Lar $\left.)\right)<r, \quad r>0$.

Taking the supremum over $t$, we get

$$
\|T u-T v\| \leq \xi(\|u-v\|)
$$

for every $u, v \in E$, with $u \geq v$. Consequently, $T$ is a partially nonlinear $\mathrm{D}$-contraction in $E$. In addition, as in the proof of Theorem 3 , we can show that the function $z$ given in condition $\left(A_{4}\right)$ achieves the inequality of the operator $z \leq T z$ in $J$. Now, we apply direction of Theorem 1 gives that the HTNFIDE (4) has a unique solution $u^{*}$, and the sequence $\left\{u_{n}\right\}$ of successive approximations introduced by Equation (9) converges monotonically to $u^{*}$.

Remark 3. The inference of Theorem 4, also still true if we substitute condition $\left(A_{4}\right)$ with $\left(B_{4}\right)$.

Example 2. Consider $J=[0,1]$ closed and bounded interval, then the HFIDE

$$
\begin{gathered}
\left(D^{\frac{3}{2}} u\right)(t)+x(t)=\frac{1}{2}\left(\tan ^{-1} u(t)+\tan ^{-1} \int_{t_{0}}^{t} \frac{(t-s)^{\frac{1}{2}}}{\Gamma\left(\frac{3}{2}\right)} g(s, u(s)) d s\right) \\
u(0)=1
\end{gathered}
$$

where $g: J \times \mathbb{R} \rightarrow \mathbb{R}$ is a function introduced by

$$
g(t, u)=\left\{\begin{aligned}
1, & \text { if } u \leq 0 \\
1+\frac{u}{1+u}, & \text { if } u>0
\end{aligned}\right.
$$

Hence, $\lambda=1, c=1, f(t, u, v)=\frac{1}{2}\left(\tan ^{-1} u+\tan ^{-1} v\right)$. Obviously, the functions $f$ and $g$ are continuous on $J \times \mathbb{R} \times \mathbb{R}$ and $J \times \mathbb{R}$, consecutively. The function $f$ achieves $\left(A_{1}\right)$ with $M_{f}=\frac{\pi}{2}$ and it is easy to prove that $g$ achieves $\left(A_{5}\right)$ with $L=1$. Further, $f(t, u, v)$ is increasing in $u$ and $v$ for any $t$ in $J$. To prove that $f$ fulfills $\left(A_{6}\right)$ on $J \times \mathbb{R} \times \mathbb{R}$, let $u_{1}, u_{2}, v_{1}, v_{2} \in \mathbb{R}$ be such that $u_{1} \geq v_{1}$ and $u_{2} \geq v_{2}$. Then,

$$
\begin{aligned}
0 \leq f\left(t, u_{1}, u_{2}\right)-f\left(t, v_{1}, v_{2}\right) \leq & \frac{1}{2}\left(\tan ^{-1} u_{1}-\tan ^{-1} v_{1}+\tan ^{-1} u_{2}-\tan ^{-1} v_{2}\right) \\
\leq & \frac{1}{2} \frac{u_{1}-v_{1}}{1+\eta_{1}^{2}}+\frac{1}{2} \frac{u_{2}-v_{2}}{1+\eta_{2}^{2}} \\
& \leq \xi_{1}\left(u_{1}-v_{1}\right)+\xi_{2}\left(u_{2}-v_{2}\right)
\end{aligned}
$$

for all $t \in J$ and for some $u_{1}>\eta_{1}>v_{1}$ and $u_{2}>\eta_{2}>v_{2}$, where $\xi_{1}$ and $\xi_{2}$ are D-functions introduced by

$$
\xi_{1}(r)=\frac{r}{1+\eta_{1}^{2}}, \text { and } \xi_{2}(r)=\frac{r}{1+\eta_{2}^{2}}
$$

for $0<\eta_{1}, \eta_{2}<r$. Furthermore,

$$
\frac{1}{2} \frac{a^{\alpha}}{\Gamma(\alpha+1)}\left(\xi_{1}(r)+\xi_{2}(a r)\right)=\frac{r}{1+\eta^{2}}<r
$$

where $\eta=\min \left\{\eta_{1}, \eta_{2}\right\}$.

At the end, the HTNFIDE (4) has a lower solution $z(t)=\frac{2}{\Gamma\left(\frac{3}{2}\right)}\left(e^{\frac{-t \frac{1}{2}}{\Gamma\left(\frac{3}{2}\right)}}-1\right)$ introduced on $J$. Thus, all the supposition of Theorem 4 are fulfilled and thus we conclude that the HTNFIDE (16) has a unique solution $u^{*}$ introduced on $J$. Moreover, the sequence $\left\{u_{n}\right\}$, introduced by 


$$
\begin{aligned}
& u_{0}(t)=z(t),
\end{aligned}
$$

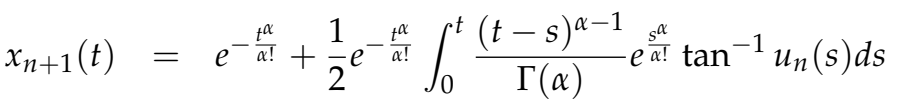

$$
\begin{aligned}
& +\frac{1}{2} e^{-\frac{t^{\alpha}}{\alpha !}} \int_{0}^{t} \frac{(t-s)^{\alpha-1}}{\Gamma(\alpha)} e^{\frac{s^{\alpha}}{\alpha !}} \tan ^{-1}\left(\int_{0}^{s} \frac{(s-\xi)^{\alpha-1}}{\Gamma(\alpha)} g\left(\xi, u_{n}(\xi)\right) d \xi\right) d s
\end{aligned}
$$

for every $t$ in $J$, converges monotonically to $u^{*}$.

\section{The First Type Linear Perturbations}

At times, it is conceivable that the non-linearity of those involved in HTNFIDE (1) does not fulfill either the supposition of Theorem 3 or the supposition of Theorem 4 . In spite of, from incising the functions $f_{1}$ and $f_{2}$ of $f$ in the form $f=f_{1}+f_{2}$ fulfill the conditions of Theorems ( 3 and 4). Consecutively. in Dhage's terminology [3], the resulting equation is said to be the hybrid integro-differential equation with the first type linear disturbance. The objective of this section is to get an existence result for such an equation.

Given above notations in the sections, we consider the nonlinear hybrid HTNFIDE

$$
\begin{aligned}
D^{\alpha} u(t)+\lambda u(t)= & f_{1}\left(t, u(t), \int_{t_{0}}^{t} \frac{(t-s)^{\alpha-1}}{\Gamma(\alpha)} g(s, u(s))\right) d s \\
& +f_{2}\left(t, u(t), \int_{t_{0}}^{t} \frac{(t-s)^{\alpha-1}}{\Gamma(\alpha)} g(s, u(s)) d s\right) \\
& u\left(t_{0}\right)=B_{0},
\end{aligned}
$$

for every $t$ in $J$, where $f_{1}, f_{2}: J \times \mathbb{R} \times \mathbb{R} \rightarrow \mathbb{R}$ and $g: J \times \mathbb{R} \rightarrow \mathbb{R}$ are continuous functions.

From a solution of the HTNFIDE (18), we denote a function $u \in C^{1}(J, \mathbb{R})$ that fulfills equation (18), where $C^{1}(J, \mathbb{R})$ is the habitual space of continuously differentiable real-valued functions introduced on on $J$.

The HTNFIDE (18) is called the hybrid fractional integro-differential equation with a the first type linear perturbation $[18,22]$.

The HTNFIDE (18) is well recognized in the literature and discussed of the existence and other properties. herein, we prove that existence of solutions by using partially compactness type conditions and the mixed partially Lipschitz.

Theorem 5. Assume that $\left(A_{1}\right)-\left(A_{3}\right)$ fulfilled with $f$ and replaced by $f_{2}$, and further let $\left(A_{1}\right),\left(A_{5}\right)$, and $\left(A_{6}\right)$ fulfilled with $f$ and replaced by $f_{1}$. If $\left(A_{7}\right)$ satisfied, then the HTNFIDE (18) has a solution $u^{*}$ introduced on $J$ and the sequence $\left\{u_{n}\right\}$ of successive approximations, introduced by

$$
\begin{aligned}
u_{1}(t)= & z(t), \\
u_{n+1}(t)= & c e^{-\lambda \frac{t^{\alpha}}{\alpha !}} \\
& +e^{-\lambda t^{\alpha} \frac{t^{\alpha}}{\alpha !}} \int_{t_{0}}^{t} \frac{(t-s)^{\alpha-1}}{\Gamma(\alpha)} e^{\lambda s^{\frac{s^{\alpha}}{\alpha !}}} f_{1}\left(s, u_{n}(s), \int_{t_{0}}^{s} \frac{(s-\xi)^{\alpha-1}}{\Gamma(\alpha)} g\left(\xi, u_{n}(\xi)\right) d \xi\right) d s \\
+ & e^{-\lambda \frac{t^{\alpha}}{\alpha !}} \int_{t_{0}}^{t} \frac{(t-s)^{\alpha-1}}{\Gamma(\alpha)} e^{\lambda \frac{s^{\alpha}}{\alpha !}} f_{2}\left(s, u_{n}(s), \int_{t_{0}}^{s} \frac{(s-\xi)^{\alpha-1}}{\Gamma(\alpha)} g\left(\xi, u_{n}(\xi)\right) d \xi\right) d s,
\end{aligned}
$$

for $t \in J$, converges monotonically to $u^{*}$. 
Proof. Set $E=C(J, \mathbb{R})$. Then, from Lemma 2 it follows that each compact chain $C$ in $E$ has the property of compatibility with respect to the order relation $\preceq$ and the norm $\|$.$\| in E$.

According to Lemma 2, the HTNFIDE (18) is equivalent to the nonlinear integral equation

$$
\begin{aligned}
u(t)= & c e^{-\lambda \frac{t^{\alpha}}{\alpha !}}+e^{-\lambda t^{\alpha} \frac{\alpha^{\alpha}}{\alpha !}} \int_{t_{0}}^{t} \frac{(t-s)^{\alpha-1}}{\Gamma(\alpha)} e^{\lambda \frac{s^{\alpha}}{\alpha !}} f_{1}\left(s, u(s), \int_{t_{0}}^{s} \frac{(s-\xi)^{\alpha-1}}{\Gamma(\alpha)} g(\xi, u(\xi)) d \xi\right) d s, \\
& +e^{-\lambda \frac{\alpha^{\alpha}}{\alpha !}} \int_{t_{0}}^{t} \frac{(t-s)^{\alpha-1}}{\Gamma(\alpha)} e^{\lambda \frac{s^{\alpha}}{\alpha !}} f_{2}\left(s, u(s), \int_{t_{0}}^{s} \frac{(s-\xi)^{\alpha-1}}{\Gamma(\alpha)} g(\xi, u(\xi)) d \xi\right) d s, \quad t \in J .
\end{aligned}
$$

Set $E=C(J, \mathbb{R})$ and introduce the operators $A$ and $B$ on $E$ by

$$
A u(t)=e^{-\lambda \frac{t^{\alpha}}{\alpha !}} \int_{t_{0}}^{t} \frac{(t-s)^{\alpha-1}}{\Gamma(\alpha)} e^{\lambda s^{\alpha} \frac{s^{\alpha} !}{\alpha}} f_{1}\left(s, u(s), \int_{t_{0}}^{s} \frac{(s-\xi)^{\alpha-1}}{\Gamma(\alpha)} g(\xi, u(\xi)) d \xi\right) d s
$$

and

$$
B u(t)=c e^{-\lambda \frac{t^{\alpha}}{\alpha !}}+e^{-\lambda \frac{t^{\alpha}}{\alpha !}} \int_{t_{0}}^{t} \frac{(t-s)^{\alpha-1}}{\Gamma(\alpha)} e^{\lambda \frac{s^{\alpha}}{\alpha !}} f_{2}\left(s, u(s), \int_{t_{0}}^{s} \frac{(s-\xi)^{\alpha-1}}{\Gamma(\alpha)} g(\xi, u(\xi)) d \xi\right) d s .
$$

Obviously, $A, B: E \rightarrow E$. In addition, HTNFIDE (18) is equivalent to the operator equation

$$
A u(t)+B u(t)=u(t), \quad t \in J .
$$

Following similar arguments to those used in the proofs of Theorems 3 and 4, we can show that operator $A$ is a nonlinear Dcontraction and partially bounded and $B$ is a partially compact operator and partially continuous in $E$. From the direct application of Theorem 2 gives that the operator equation $u \leq A u+B u$ has a solution $u^{*}$. Thus, HTNFIDE (18) has a sequence $\left\{u_{n}\right\}_{n=1}^{\infty}$, and the solution $u^{*}$ introduced by (19) monotonic converges to $u^{*}$. This completes the proof.

The inference of Theorem 5 residues true if we substitute $\left(A_{7}\right)$ with $\left(B_{7}\right)$. The HTNFIDE (18) has a upper solution $y \in C^{1}(J, \mathbb{R})$.

Example 3. On the interval $J=[0,1]$ we consider the HTNFIDE,

$$
\begin{aligned}
D^{\frac{1}{2}} u(t)+u(t) & =\tan ^{-1} u(t)+\tanh \left(\int_{0}^{t} \frac{(t-s)^{\frac{-1}{2}}}{\Gamma\left(\frac{1}{2}\right)} \sinh u(s) d s\right), \\
u(0) & =1 .
\end{aligned}
$$

If we apply the Theorem 5, we will get $\lambda=1, c=1, f_{1}(t, u, v)=\tan ^{-1} u, f_{2}(t, u, v)=\tanh v$ and $g(t, v)=\sinh v$. Therefore the function $f_{1}$ fulfills $\left(A_{1}\right)$ with $M_{f_{1}}=\frac{\pi}{2}$ and also fulfills $\left(A_{6}\right)$ with $\xi_{1}(r)=\frac{r}{1+\xi^{2}}, 0<\xi<r$ and $\xi_{2}(r)=0$. Now $f_{2}$ fulfills $\left(A_{1}\right)$ with $M_{f_{2}}=1$ and it is increasing in $v$, thus $\left(A_{2}\right)$ fulfills. Likewise, $g$ fulfills $\left(A_{3}\right)$. In the end, $z(t)=3\left(e^{-t}-1\right)$, for every $t \in J$, is a lower solution of the HTNFIDE (24) on $J$, and thus $\left(A_{7}\right)$ is fulfilled. Next, by Theorem 5 , the HTNFIDE (24) possesses a solution $u^{*}$ on $J$, and the sequence $\left\{u_{n}\right\}_{n=1}^{\infty}$, introduced by

$$
\begin{aligned}
u_{1}(t)= & 3\left(e^{-t}-1\right), \\
u_{n+1}(t)= & e^{-\frac{t^{\alpha}}{\alpha !}}+e^{-\frac{t^{\alpha}}{\alpha !}} \int_{t_{0}}^{t} \frac{(t-s)^{\alpha-1}}{\Gamma(\alpha)} e^{s^{\alpha} !} \tan ^{-1} u_{n}(s) d s \\
& \left.+e^{-\frac{t^{\alpha}}{\alpha !}} \int_{0}^{t} \frac{(t-s)^{\alpha-1}}{\Gamma(\alpha)} e^{s^{\alpha}} \frac{\sinh }{\alpha !} \tan \sinh u_{n}(\xi) d \xi\right) d s,
\end{aligned}
$$

for $t \in J$, converges monotonically to $x^{*}$. 


\section{Conclusions}

From our above study, we observe that if the HTNFIDE ((1) or (18)) have an upper $y$ solution, then a lower solution $z$ such that $y \geq z$, follows the congruous solutions $u_{*}$ and $u^{*}$ of the HTNFIDE ((1) or (18)) that fulfills $u_{*} \leq u^{*}$, and these are the maximum and minimum solutions in a vector segment $[z, y]$ of the Banach space $E=C(J, \mathbb{R})$. In fact, the order relation $\preceq$ introduced by Equation (5) is equivalent to the order relation introduced by

$$
K=\{u \in C(J, \mathbb{R}) \mid u(t) \geq 0, \text { for every } t \in J\}
$$

which is a closed set in $C(J, \mathbb{R})$. We also observe that the Dhage iteration method is a useful tool for proving existence results for certain nonlinear hybrid fractional integro-differential equations. The HTNFIDE is considered here, for which we have illustrated the Dhage iteration method to obtain algorithms for the solutions under weaker partially Lipschitz and compactness conditions, is of fairly simple nature. An analogous study could also be made for more complicated fractional integro-differential equations on using a similar approach with appropriate modifications. However, we left the study along this direction to future work.

Author Contributions: Conceptualization, A.K.; Methodology, F.H.D. and A.K.; Supervision, F.H.D. and A.K.; Writing-original draft, A.K. and A.T.A.-A.; Writing—review and editing, A.K. All authors have read and agreed to the published version of the manuscript.

Funding: The payment of the APC will be covered by the Fundamental Research Grant Schemes which is having Ref. No.: FRGS/1/2018/STG06/UPM/01/3 and vot number 5540153.

Acknowledgments: The authors would like to thank the referees and Editors for the useful comments and remarks, which improved the present manuscript substantially. The authors are also very grateful to the Ministry of Education Malaysia for the financial support that will cover the APC by using the the Fundamental Research Grant Scheme (FRGS) under Ministry of Education with project number FRGS/1/2019/STG02/UPM/02/3.

Conflicts of Interest: The authors declare no conflict of interest.

\section{References}

1. Sitho, S.; Sotiris K. Ntouyas and Jessada Tariboon. Existence results for hybrid fractional integro-differential equations. J. Bound. Value Probl. 2015, 2015, 113. [CrossRef]

2. Dhage, B.C.; Khurape, G.T.; Shete, A.Y.; Salunkhe, J.N. Existence and Approximate Solutions for Nonlinear Hybrid Fractional Integrodifferential Equations. Int. J. Anal. Appl. 2016, 11, 157-167.

3. Dhage, B.C.; Dhage, S.B.; Graef, J.R. Dhage iteration method for initial value problems for nonlinear first order hybrid integrodifferential equations. J. Fixed Point Theory Appl. 2016, 18, 309-326. [CrossRef]

4. Ardjouni, A.; Djoudi, A. Approximating Solutions of Nonlinear Hybrid Caputo Fractional Integro-Differential Equations via Dhage Iteration Principle. Ural. Math. J. 2019, 5, 3-12. [CrossRef]

5. Martínez-García, M.; Gordon, T.; Shu, L. Extended Crossover Model for Human-Control of Fractional Order Plants. IEEE Access 2017, 5, 27622-27635. [CrossRef]

6. Martínez-García, M.; Zhang, Y.; Gordon, T. Memory Pattern Identification for Feedback Tracking Control in Human-Machine Systems. Hum. Factors J. Hum. Factors Ergon. Soc. 2019. [CrossRef] [PubMed]

7. Martínez-García, M.; Zhang, Y.; Gordon, T. Modeling Lane Keeping by a Hybrid Open-Closed-Loop Pulse Control Scheme. IEEE Trans. Ind. Inform. 2016, 12, 2256-2265. [CrossRef]

8. Kılıçman, A.; Damag, F.H.M. Some Solution of the Fractional Iterative Integro-Differential Equations. Malays. J. Math. Sci. 2018, 12, 121-141.

9. Kreyszig, E. Introductory Functional Analysis with Applications; Wiley: New York, NY, USA, 1978.

10. Damag, F.H.; Kılıçman, A.; Dutta, H.; Ibrahim, R.W. A Note on the Lower and Upper Solutions of Hybrid-Type Iterative Fractional Differential Equations. Natl. Acad. Sci. Lett. 2019, 1-5. [CrossRef]

11. Zeidler, E. Nonlinear Functional Analysis and Its Applications: II/B: Nonlinear Monotone Operators; Springer Science \& Business Media: Berlin/Heidelberg, Germany, 2013. 
12. Damag, M.F.H. Existences and Uniqueness of Solutions for Some Classesof Iterative Fractional Functional Integral Differential Equations (2017). Available online: http://psasir.upm.edu.my/id/eprint/68642 (accessed on 1 April 2020).

13. Guo, B.; Pu, X.; Huang, F. Fractional Partial Differential Equations and Their Numerical Solutions; World Scientific: Singapore, 2015.

14. Diethelm, K. The Analysis of Fractional Differential Equations: An Application-oriented Exposition Using Differential Operators of Caputo Type; Springer Science and Business Media: Berlin/Heidelberg, Germany, 2010.

15. Lakshmikantham, V.; Vatsala, A.S. Basic theory of fractional differential equations. Nonlinear Anal. Theory Methods Appl. 2008, 69, 2677-2682. [CrossRef]

16. Podlubny, I. Fractional Differential Equations: An Introduction to Fractional Derivatives, Fractional Differential Equations, to Methods of Their Solution and Some of Their Applications; Elsevier: Amsterdam, The Netherlands, 1998.

17. Lakshmikantham, V. Monotone Iterative Techniques for Discontinuous Nonlinear Differential Equations; Routledge: Abingdon-on-Thames, UK, 2017.

18. Dhage, B.C. Hybrid fixed point theory in partially ordered normed linear spaces and applications to fractional integral equations. Differ. Equ. Appl. 2013, 5, 155-184. [CrossRef]

19. Dhage, B.C. Operator theoretic techniques in the theory of nonlinear hybrid differential equations. Nonlinear Anal. Forum 2015, 20, 15-31.

20. Dhage, C.B. Partially condensing mappings in partially ordered normed linar spaces and applications to functional integral equations. Tamkang J. Math. 2014, 4, 397-426. [CrossRef]

21. Dhage, B.C.; Dhage, S.B. Approximating solutions of nonlinear first order ordinary differential equations. Glob. J. Math. Sci. 2015, 2, 25-35.

22. Dhage, B.C. Global attractivity and stability results for comparable solutions of nonlinear fractional integral equations. Differ. Equ. Appl. 2014, 6, 165-186.

(C) 2020 by the authors. Licensee MDPI, Basel, Switzerland. This article is an open access article distributed under the terms and conditions of the Creative Commons Attribution (CC BY) license (http:/ / creativecommons.org/licenses/by/4.0/). 